scheidungslogik zwischen alternativen Lösungen herausgelöst.

Der VDI-Report 29 kann zu einem Preis von DM 18,-- bezogen werden vom Verein Deutscher Ingenieure, VDI-Hauptgruppe, GrafRecke-Straße 84, D-40239 Düsseldorf, Fax: 0211/6214-148, e-mail: brennecke@vdi.de. Der Tagungsband wird im Verlag edition sigma erscheinen.

》

\section{Bericht über den 5. Weltkon- greß "Intelligent Transport Systems" in Seoul, Korea}

\section{Konferenzbericht von Günter Halbritter und Torsten Fleischer, ITAS}

Die international wohl bedeutendste Jahrestagung zum Einsatz von Informations- und Kommunikationstechniken (IuK-Techniken) im Verkehr, der 5. Weltkongreß "Intelligent Transport Systems" vom 12.-16. Oktober in Seoul/Korea, war durch eine Vielzahl interessanter Einzelergebnisse in diesem aus seiner ersten stürmischen Entwicklungsphase herausgetretenen Technikbereich geprägt, wenngleich herausragende Ereignisse fehlten. Insgesamt stellte auch diese ITS-Konferenz, wie auch bereits ihre Vorgänger, mit drei Plenary Sessions, 12 Executive Sessions mit 72 Beiträgen, 28 Special Sessions mit 140 Beiträgen und 104 Technical Sessions mit 520 Beiträgen eine Mammutveranstaltung dar. Mehr als die Hälfte der technischen Konferenzbeiträge stammte aus Korea und dem übrigen asiatisch-pazifischen Raum (hier v.a. aus Japan). Zeitgleich mit der Konferenz fand eine Ausstellung zum praktischen Einsatz von IuK-Techniken im Verkehrsbereich statt. Diese wurde hauptsächlich von asiatischen Ausstellern bestritten. Im Unterschied zur Vorgängerveranstaltung in Berlin, bei der alle Regionen in großem Umfang vertreten waren, war die Präsenz amerikanischer und europäischer Hersteller auf dieser Ausstellung eher gering. Die deutsche Industrie wurde lediglich durch die Unternehmen Bosch-Signalbau Huber und Dr. Seufert GmbH Karlsruhe repräsentiert.

In diesem Bericht sollen einige Einschätzungen zu dieser technisch-wissenschaftlichen
Großveranstaltung gegeben werden. Einschränkend muß bemerkt werden, daß eine solch umfangreiche Veranstaltung mit bis zu zwölf parallel laufenden Sitzungen auch von zwei Konferenzteilnehmern, die zudem noch eigenen Vortragsverpflichtungen nachkommen mußten, nicht zu leisten war. Neben einer allgemeinen, notwendigerweise subjektiven Einschätzung zu Ablauf und inhaltlicher Schwerpunktsetzung des Gesamtkongresses, beschränkt sich die Darstellung auf einige ausgewählte Sitzungen, wobei als Auswahlkriterium der Einsatz von IuK-Techniken in Ballungsräumen diente. Vorliegender Bericht entspricht somit nicht dem Anspruch auf eine umfassende und objektive Berichterstattung zum Kongreß.

Die Tagung stand insgesamt ganz im Zeichen des Straßenverkehrs, öffentliche Verkehrsträger spielten nur eine untergeordnete Rolle. Weiterhin war die Tagung sehr von technischen Beiträgen geprägt, die zum großen Teil aus dem asiatisch-pazifischen Raum stammten. Beiträge, die sich mit der Umsetzung dieser Techniken beschäftigten, waren deutlich in der Minderheit, entsprechend auch Wirkungs- und Folgenanalysen dieser Techniken. Diesbezügliche Ansätze waren zwar in manchen Beiträgen - vor allem von europäischen und australischen(!), in geringerem Umfang auch von nordamerikanischen Referenten - zu finden, zeigten aber auch hier häufig die hinlänglich bekannten Defizite bezüglich empirischer Fundierung und Übertragbarkeit. Festzustellen ist, daß Problembewußtsein in bezug auf Forschungsbedarf $\mathrm{zu}$ Wirkungen und Folgen von IuK-Techniken im Verkehr hauptsächlich von Vertretern aus Ländern mit vergleichsweise geringer (oder nur punktueller) industrieller Aktivität in der Verkehrstelematik signalisiert wurde (etwa die skandinavischen Länder und die Niederlande, aber auch Australien und Neuseeland), während seitens der Vertreter der großen "Forschungsnationen" eher das Herausstellen von Erwartungen und Hoffnungen dominierte.

Inhaltlich prägend für die Konferenz waren unserer Einschätzung nach letztlich die Beiträge aus dem anglo-amerikanischen Raum, obwohl der Anteil technischer Beiträge aus dem Bereich der europäischen Union fast doppelt so hoch war wie der aus den USA (140 zu 79). Ein Grund für diese Einschätzung ist sicherlich die konzeptionelle Klarheit, die insbesondere die 
US-amerikanischen Beiträge auszeichnete. Hierzu trug wohl auch die im Juni dieses Jahres erfolgte Verabschiedung des "Public Law 105178 - Transport Equity Act for the 21. Century (TEA21)" bei. Dies gilt als wichtigster gesetzgeberischer Akt im Bereich des bodengebundenen Verkehrs in den letzten Jahren und baut auf auf den Regelungen des Intermodal Surface Transportation Efficiency Act von 1991. Neben der Regelung der Finanzierung von Infrastruktur-, Verkehrs- und Forschungsprojekten im bodengebundenen Verkehr schafft TEA21 auch eine verbindliche Rechtsgrundlage für die bundesweite Einrichtung von Telematikdiensten. Dieses Gesetz regelt nicht nur die Finanzierung dieser Dienste, sondern auch wichtige Standardsetzungen bundesweit einheitlich. Es wird $\mathrm{zu}$ prüfen sein, inwieweit diese gesetzlichen Regelungen tatsächlich den genannten hohen Erwartungen genügen und ob sie Vorbildcharakter für andere Staaten besitzen.

Der finanzielle Rahmen, der mit TEA21 von seiten des Bundes für ITS-Programme im Zeitraum von 1998-2003 zur Verfügung steht, umfaßt insgesamt 1,282 Mrd \$. Davon sind 603 Mio. \$ für Forschung, Feldversuche, Ausbildung und Standardentwicklung vorgesehen. Der wesentliche Restbetrag wird für Programme zur Anwendung von ITS-Techniken und zum Aufbau entsprechender Infrastruktur (darunter 482 Mio. \$ für Ballungsräume und 184 Mio. \$ für ländliche Regionen) eingesetzt. Darüber hinaus werden ITS-Aktivitäten auch aus weiteren Quellen gefördert, so stehen auch Mittel aus Umweltschutzfonds zur Verfügung, falls ITSMaßnahmen zur Verbesserung des Verkehrsflusses und damit zur Verbesserung der Luftqualität beitragen.

TEA21 verlangt die Entwicklung von Richtlinien für die Beschaffung und für die unabhängige Bewertung von ITS-Software, sowie die Durchführung von Lifecycle-KostenAnalysen für die geförderten Programme. Alle geförderten Programme müssen mit der nationalen Architektur und den entsprechenden Standards übereinstimmen. Diese Standards sollen bis Juni 1999 bzw. Januar 2001 vorliegen.

Im einzelnen soll nachfolgend über einige ausgewählte Sitzungen berichtet werden, die für die Thematik "Mobilität in Ballungsräumen" von besonderer Bedeutung sind.

\section{Public Private Partnership (Executive Sessi- on 6)}

Die Sitzung "Public-Private Partnership" stand im Zeichen anspruchsvoller Zielvorstellungen und der bisherigen praktischen Erfahrungen dieses Organisationsmodells. Die vorgetragenen Erfahrungen aus asiatischen, US-amerikanischen und englischen Projekten erfüllten diese Erwartungen nur bedingt. Wolfgang Hahn, BMV, unterstrich als Moderator dieser Sitzung die Bedeutung dieses Organisationsmodells für die erfolgreiche Einführung von Telematikdiensten.

Dennis L. Foderberg stellte das bereits 1991 begonnene ITS-Programm in Minnesota/USA, Minnesota Guidestar, ein US-amerikanisches Vorzeigeprojekt, vor. Hervorzuheben ist dabei, daß in diesem Projekt nicht nur der Straßenverkehr, sondern auch der öffentliche Verkehr berücksichtigt werden. Das Programm umfaßt 60 Partnerschaften mit über 70 Partnern aus dem öffentlichen, privaten und wissenschaftlichen Bereich. Dabei wurden über 90 Mio.\$ investiert. Der Schwerpunkt der Programme bezieht sich auf die Bereitstellung von Verkehrsinformationen und die Verbesserung des Verkehrsmanagements in dem von den beiden Städten Minneapolis und St. Paul gebildeten Ballungsraum. Nach den Ausführungen von Foderberg wurde in dem Verkehrskorridor zwischen den beiden Städten ein Verkehrsmanagement unter Einbeziehung von örtlichen Straßen realisiert, bei dem neben technischen eine Reihe verwaltungstechnischer Fragen gelöst werden mußten. Hervorzuheben sind der durch ein Verkehrsmanagementzentrum gesteuerte Verkehrsfluß, einschließlich des Einsatzes von Zufahrtsbeschränkungen (ramp-metering). Mit dem Teilprojekt Travlink wurde die Verkehrsinformation im Bereich des öffentlichen Verkehrs so verbessert, daß für Pendler ein Anreiz geschaffen wurde, verstärkt öffentliche Verkehrsmittel zu nutzen.

Die Ausführungen von Michael Della Rocca, Quade \& Douglas Inc., gaben einen Überblick zu Konzeption, zu den institutionellen Bedingungen und zu einigen Fallstudien zum Einsatz von ITS im Verkehr des Großraums New York. Außer der klaren Strukturierung des technischen und organisatorischen Aufwandes auf den Ebenen der Kommunikati- 
on, des Transports und der institutionellen Einbettung der Dienste wurden auch Finanzierungskonzepte für diese Dienste angesprochen. Die neuen Rollen der verschiedenen an den Projekten beteiligten privaten und öffentlichen Partner wurden an Hand einer übersichtlichen Aufgabenteilung dargestellt. Dabei wurde deutlich, daß die öffentlichen Partner die Voraussetzung für die Entwicklung neuer Märkte mit Gewinnchancen schaffen müssen. Auch bei den Projekten im Großraum New-York werden fortgeschrittene öffentliche Verkehrssysteme berücksichtigt.

Im Mittelpunkt der Fallstudie 1 - NYC Model Deployment Initiative (MDI) - steht die Informationsbereitstellung zur intermodalen Routenplanung unter Berücksichtigung von Echtzeitdaten. Die Fallstudie 2 - New Jersey Transportation Partnership Program - bezieht sich auf das Engagement des privaten Sektors bei der Planung, dem Entwurf, der Konstruktion, der Finanzierung und des Betriebs von Verkehrsprojekten. Hervorzuheben ist dabei das "Route 80 Magic Project (MAGIC- Metropolitan Area Guidance Information and Control)", das als Pilotprojekt für den Einsatz verschiedener Dienste ausgewählt wurde.

\section{International ITS Standards: Is the Process Working? (Special Session 1)}

Referenten bei dieser Session waren Martin Rowell (Navigation Technologies, Chairman ISO TC 204), Teiji Akiyama (AIST/MITI) und Gé van Toorenburg (Rijkswaterstaat) (Niederländisches Verkehrsministerium, Chairman CEN TC 278).

Übereinstimmend wurde festgestellt, daß Standardisierungsaktivitäten von der absoluten Mehrheit der im Telematikbereich aktiven Unternehmen zwar als wichtig eingestuft, jedoch von diesen - bis auf Ausnahmen - weder personell noch finanziell ausreichend unterstützt würden. Die Interpretation der Ursachen ging auseinander, neben zu kurzfristigen Planungsansätzen im Management (Stichwort: Shareholder Value-Denken) wurden auch bisher nicht nachgewiesene Profitabilität von TelematikTechniken und unternehmensstrategische Gründe angeführt, wie zum Beispiel das Schaffen proprietärer (von Normungsgremien unabhängiger) "de facto"-Standards durch Unter- nehmen oder Konsortien, etwa auch der von deutschen Unternehmen verfolgte GATS (Global Automotive Telematics Standard)-Ansatz.

Finanzielle Unterstützung und personelle Beteiligung durch Regierungen wurde übereinstimmend gewünscht. Vor allem sollten sich staatliche Stellen, die ja mittels Telematiktechniken durchaus auch verkehrspolitische Ziele umsetzen wollen, an der Systemdefinition solcher Techniken beteiligen.

Weitgehend klar ist, was Gegenstand von Standardisierungsanstrengungen sein sollte. Der zügige Abschluß von Standardisierungsarbeiten ist aber eher die Ausnahme. Für internationale (ISO-)Standards wurde ein durchschnittlicher Zeitraum von 6,5 Jahren von der Aufnahme der Arbeiten bis zum Inkrafttreten eines Standards genannt. Angesichts des Entwicklungstempos vor allem im IuK-Bereich gilt dies als deutlich zu lang.

Nationale Standardisierungsarbeiten (USA, Japan) werden in der Regel schneller abgeschlossen als regionale (Europa!), dafür tun sich diese sogenannten "island nations" bei der Kompromißfindung auf internationaler Ebene letztlich schwerer als die Europäer, die zumindest einen Teil dieses Prozesses schon hinter sich haben. Da die internationale Standardisierung nicht zuletzt deshalb als zu langwierig gilt, scheint es eine Tendenz zu geben, vor allem die regionale Standardisierung voranzutreiben.

\section{Deployment: Initiatives for ITS in Cities (Special Session 6)}

Bemerkenswert bezüglich der Deutlichkeit der verkehrspolitischen Aussagen war der Vortrag von Dr. Wim A.G. Blonk, Direktor DGVII, EU-Kommission, der die Rolle der EU bei der Einführung von IuK-Techniken im Ballungsraumverkehr in Europa behandelte. Den Schlüsselbegriff der europäischen Verkehrspolitik solle "sustainable mobility" darstellen. Um diese $\mathrm{zu}$ erreichen, sind nicht nur die Marktkräfte weiter zu entwickeln, wozu auch verbesserte Systeme des Verkehrsmanagement und der Gebührenerhebung beitragen können, sondern auch Konzepte für eine bessere Steuerung der Nachfrage im Mobilitätsbereich. Diese solle u.a. die Bereitstellung von multimodalen Verkehrsinformationen umfassen, die als Voraussetzung für die Verlagerung von Stra- 
Benverkehr auf öffentliche Verkehrsträger angesehen wird. Weiterhin solle die Etablierung fairer und effizienter Preise für alle Verkehrsträger zur Zielerreichung beitragen. Weitere Schwerpunkte der EU-Verkehrspolitik sind die Verbesserung der Verkehrssicherheit, speziell im Straßenverkehr, und Verminderung der Umweltauswirkungen des Verkehrs, wobei neben den $\mathrm{CO}_{2}$-Emissionen den Dieselrußemissionen eine besondere Bedeutung zukommt.

Ein wesentlicher Schwerpunkt der EUVerkehrspolitik wird in der Konzipierung und Umsetzung politischer Rahmenbedingungen gesehen, die eine Entwicklung zu nachhaltigen, integrierten Verkehrssystemen sicherstellen. Weiterhin sollen im Rahmen der EU-Arbeiten praktische Werkzeuge entwickelt werden, die sowohl von Verkehrsdiensteanbietern wie auch von politischen Entscheidern genutzt werden können.

Als wichtige Anwendungen für IuK-Techniken im städtischen Verkehr werden die folgenden angesehen:

- Straßenbenutzungsgebühren

- Zugangskontrolle

- Routenführung

- Verkehrsmanagement

- Parkraummanagement

- Elektronische Buchungs- und Zahlungssysteme

- Sicherstellung der Interoperabilität der Systeme.

Um die städtischen Verkehrssysteme nachhaltiger zu gestalten, werden die folgenden Maßnahmen vorgeschlagen:

- Steigerung der Qualität und Erreichbarkeit öffentlicher Verkehrsträger

- Reduktion der Verkehrsnachfrage

- Attraktivitätssteigerung von Zufußgehen und Fahrradfahren

- Sicherstellung einer hohen Lebensqualität ohne Abhängigkeit vom Auto

- Aktives Straßenverkehrsmanagement in staugefährdeten Gebieten

- Berücksichtigung des Verkehrs bei der Entwicklung von Strategien der räumlichen, ökonomischen und sozialen Entwicklung

- Flexible Arbeitszeiten

- Entwicklung von Tür zu Tür - Verkehrssystemen.
Im anschließenden Gespräch bestärkte Blonk nochmals die verkehrspolitischen Intentionen der Kommission und wies dabei auch auf das inzwischen vorliegende Weißbuch "Faire Preise für die Infrastrukturbenutzung: Ein abgestuftes Konzept für einen Gemeinschaftsrahmen für Verkehrsinfrastrukturgebühren in der EU" hin, in dem auf die Vielzahl der Systeme zur Anlastung der Verkehrskosten in Europa hingewiesen wird, die zu erheblichen Wettbewerbsverzerrungen führen und darüber hinaus Anreize zur Verringerung von Umweltkosten und effizientere Infrastrukturpolitik beschneiden. Es werden daher Grundsätze zur schrittweisen und progressiven Harmonisierung der Gebührenberechnung für alle wichtigen gewerblichen Verkehrsarten entwickelt.

\section{Challenges of Urban Growth (Special Sessi- on 17)}

Diese Sitzung war eine der wenigen, die sich mit den Folgen des wachsenden Einsatzes von Telematikdiensten auf die Lebensbedingungen in Ballungsräumen auseinandersetzte.

Eric Sampson, UK Department of Environment, Tranport and Regions, London, gab einen Überblick über die verkehrspolitische Grundsatzerklärung der englischen Regierung "The Government's White Paper on the Future of Transport". Dieser Bericht enthält eine bemerkenswert kritische Analyse der Verkehrssituation und der absehbaren weiteren Entwicklung des Verkehrs, insbesondere des Straßenverkehrs, wegen der wachsenden Belastungen für die Gesundheit der Menschen und für die lokale, regionale und globale Umwelt sowie wegen der wachsenden Ineffizienzen durch Staus und Engpässe. Der Bericht fordert daher einen "New Deal for transport", der sich in einer integrierten Verkehrspolitik wiederfinden soll. Integration ist dabei nicht nur im engen Sinne einer technischen Integration der verschiedenen Verkehrsträger gemeint, sondern im Sinne einer Integration verschiedener für die Verkehrspolitik bedeutender Politikbereiche, wie Raumplanung, Erziehung und Umwelt.

Das "White Paper" stützt sich auf die Ergebnisse einer Reihe wissenschaftlicher Beratungsorgane, wie der "Royal Commission on Environmental Pollution", die bereits 1994 eine kontinuierliche, jährliche Steigerung der Mine- 
ralölsteuer forderte. Diese Forderung wird im UK auch bereits umgesetzt, mit dem Ergebnis, daß die jährliche Steigerung der Mineralölsteuer um 5\% zu Kraftstoffpreisen geführt hat, die erheblich über denen in Deutschland liegen. Im UK haben Benzin und Dieselkraftstoff in etwa den gleichen Preis, somit liegt dort keine indirekte Subventionierung des LKW-Verkehrs vor.

Sehr deutliche Worte zur Ambivalenz der ITS-Techniken in bezug auf die Erreichung verkehrspolitischer Ziele fand Milton D. Harmelink, Ontario, Kanada, in seinem Vortrag "Sustainable Urban Mobility and ITS". Harmelink führte aus, daß rein technische Maßnahmen zur Lösung der anstehenden Transportprobleme nur bedingt beitragen können, daß es vielmehr darum gehe, technische Innovationen in politische Rahmenbedingungen und zielorientierte Handlungstrategien einzubetten. ITS-Techniken können somit zur "nachhaltigen Mobilität" beitragen, dies geschieht jedoch weder automatisch noch notwendigerweise. Harmelink betonte, daß beim Ballungsraumverkehr im Vergleich zum Regionalverkehr eine erheblich kompliziertere Situation vorliege. Die Vorteile eines verbesserten Straßenverkehrs, wie sie in vielen Kosten-Nutzen-Analysen zu Straßenbauprojekten nachgewiesen würden, beruhten auf rechnerischen Zeitersparnissen sowie reduzierten Kraftstoffverbräuchen und Emissionen aufgrund der verbesserten Verkehrsbedingungen. Diese Rechenergebnisse besäßen jedoch nur begrenzte Gültigkeit, da sie von fixiertem Transportaufkommen ausgingen und langfristige Rückkopplungseffekte außer Acht lassen. Harmelink verwies auf eine Reihe empirischer Untersuchungen, die zeigten, daß Verbesserungen der Automobilität immer nur die Balance zu einer höheren Nachfrage des Automobilverkehrs verschoben hätten. In eine ähnlich Richtung könne auch der Einsatz von ITS-Techniken wirken, wenn er nicht mit eindeutigen Regelungen zur Verbesserung der Gesamtverkehrssituation verbunden sei. Als ITS-Anwendungen mit positiven Auswirkungen für das Gesamtverkehrssystem seien die folgenden anzusehen:

- Verkehrsüberwachung mit Prioritätengewährung für mehrfach besetzte Fahrzeuge,

- Verkehrsinformation für alle Verkehrssysteme eines Ballungsraums mit Angabe der Transitmöglichkeiten,
- Verkehrsmanagement mit Berücksichtigung des "congestion pricing".

ITS-Systeme können somit als Instrument zur Erreichung der gesellschaftlich gewünschten Balance zwischen Individualverkehr und öffentlichem Verkehr angewandt werden, sie können jedoch auch der Erreichung einer "nachhaltigen Mobilität" entgegenstehen.

\section{MoTIV - Mobility in Transport in Intermodal Traffic (Special Session 21)}

Die Session fand vor ca. 40 Teilnehmern statt. Gegenstand der Session war die Vorstellung des Konzeptes des Programmes MoTiV sowie von in diesem Rahmen durchgeführten Einzelarbeiten. Vortragende in dieser rein von deutschen Referenten bestrittenen Veranstaltung waren Armin Anders (SIEMENS AG), Karin Hempel (Robert Bosch GmbH Hildesheim), Thomas Krist (debis-Systemhaus) und Boris Kerner (Daimler-Benz AG).

Die beiden ersten Vortragenden haben versucht, die MoTiV-Arbeiten im Rahmen eines problemorientierten Ansatzes vorzustellen. Anders gab einen Überblick über das Konzept und die laufenden Tätigkeiten im Programm, Karin Hempel stellte die Arbeiten im Mobilitäts- und Transport-Verbund (MTV) vor. Die Vorträge 3 (zur Systemarchitektur des Personal Traveler Assistant (PTA)) und 4 (zu Ansätzen und Problemen der Verkehrssimulation) waren hingegen eher theoretisch-technisch orientiert.

Etwas polemisch-plakativ zusammengefaßt konnte beim wenig vorinformierten Zuhörer der Eindruck entstehen, daß der Hauptinhalt des MoTiV-Programmes die Entwicklung eines PTA sein muß, wobei der Systemarchitektur des PTA und den Simulationstools besondere Bedeutung zukommt.

Bedauerlicherweise gelang es dem kurzfristig als Vertreter eingesprungenen Chairman nicht, die thematisch recht unterschiedlichen Vorträge in einen inhaltlichen Zusammenhang zu stellen oder in Bezug zum Programm zu setzen. Zudem wurden im Konferenzprogramm - im Unterschied zu anderen Sessions - keine Angaben zum Inhalt dieser Session gemacht, wodurch zumindest nichteuropäischen Konferenzteilnehmern damit der Inhalt weitgehend verschlossen geblieben sein dürfte. Ein Indiz dafür war die "Europalastigkeit" des Publikums. 